\title{
SYNTHESIS OF NONLINEAR CHARACTERISTICS FOR THE MOBILE ROBOT CONTROL SYSTEM
}

\author{
VASILIY BERDNIKOV and VALERIY LOKHIN \\ Department of Control Problems, MIREA - Russian Technological University, 78 Vernadsky Avenue \\ Moscow, 119454, Russia \\ E-mail: berdnikov_vp@mail.ru
}

\begin{abstract}
The article proposes a methodology for the synthesis of nonlinear controllers for a mobile robot. The new approach improving quality indicators of the system is presented. Control actions are calculated on the basis of mesh-free approximation of a value function corresponding optimal control or differential game problem. Due to computational efficiency it is possible to apply the proposed method for real-time applications. This approach assumes that the control system includes a block for processing complete information about the mobile robot. The paper shows the superiority of quality indicators in comparison with the classical methods of synthesis of controllers for a mobile robot. The article ends with an analysis of the influence of various integral criteria on the speed and overshoot of the control system.
\end{abstract}

\section{Introduction}

Mobile robotics is an area of active scientific research [1-4]. This is primarily due to the fact that mobile robots (MR) are finding more and more applications in the civil and special spheres of human life. Recent decades have been characterized by the use of artificial intelligence methods (in particular, fuzzy logic) for MR control tasks [5-8]. The issues of stability and the choice of the optimal structure of an intelligent fuzzy controller as well as its parameters tuning are the key in this direction.

In practice, the theory of absolute stability is used as a convenient tool for studying the stability of intelligent control systems. This approach allows to determine acceptable sectors in which non-linear characteristics of the conceptual (fuzzy) controller can be located. However, in most cases, the classical methods of the theory of absolute stability are insufficient and do not allow the determination of the complete boundaries of sectors. To overcome this obstacle, a number of numerical algorithms were proposed for constructing Lyapunov functions, which solve the absolute stability problem of a system with several non-stationary elements (see, for example, [9]). In [10, 11], these algorithms were applied to construct guaranteed stability regions of automatic control system (ACS) with various types of controllers and parametrically disturbed objects.

Further development of the algorithms given above is possible due to the application of the theory of optimal control and differential games. Well known result of these theories states that optimal control actions can be computed using value function. Since in most cases the analytical solutions can't be found, the number of numerical algorithms was developed (see for example [12]). These algorithms have proven convergence, but cost a lot of computer memory and CPU time. In this paper we introduce a new approach, which uses mesh-free methods for value function approximation [13, 14]. As shown below, obtained approximations have a small evaluation time and can be applied in real-time applications. Moreover, value functions are closely related to Lyapunov functions and in the case when some additional requirements are satisfied, the resulting system is asymptotically stable $[15,16]$.

When the structure of controller is known, it is possible to synthesize nonlinear controller which parameters depend on MR state vector. This requires the inclusion of block processing 
the vectors of full information about the state of mobile robot. The latter aspect represents a great practical interest for organizing the control of a highly maneuverable object that has (or permits) full state information. For example, in the task of trajectory control of a mobile robot in plane, full information refers to the vector consisting of Cartesian coordinates and the angular orientation of the robot. In most cases such information can be obtained by installing appropriate on-board sensors for mobile robot. The main purpose of this paper is to show that the use of complete information with the proposed synthesis method can significantly improve the quality indicators of the trajectory motion of MR.

The article is structured as follows. Section 2 gives a complete description of the solving problem. Also, the equations of MR motions and the structure of the ACS is given. In Section 3 , the parameters of the controller with constant coefficients are synthesized, followed by the graphs of MR positioning errors during trajectory motion. Section 4 is devoted to the synthesis of nonlinear characteristics of the controller in the presence of complete information about the MR state vector and the given integral quality criterion. Comparisons are made with the results from Section 3. An analysis of the influence of the integral criterion on the quality of trajectory motion of the MR is presented in Section 5. A summary of the results and conclusion are given in Section 6.

\section{Problem Formulation}

A two independent drive wheels mobile robot can be considered as a moving object with a high degree of controllability. The expediency of independent rear wheels for this type of MR was shown in [17] (Figure 1). This configuration increases maneuverability, but at the same time it requires a special approach to the synthesis of nonlinear characteristics of the controller, providing not only stability, but also the required quality.

For simplicity, we assume that there are no non-stationary parameters in the MR. The kinematic equations of such MR have the following form [18]:

$$
\begin{aligned}
& \dot{x}=V \cos (\theta) \\
& \dot{y}=V \sin (\theta) \\
& \dot{\theta}=\omega
\end{aligned}
$$

One of the most common methods of trajectory control MR is based on the concept of tracking a referenced vehicle (RV). In this case, the RV implements the ideal movement along the desired trajectory, and the MR seeks to minimize the deviation from this movement (Figure $2)$.

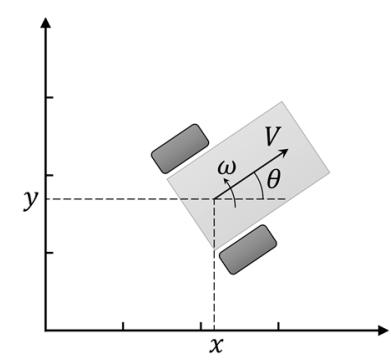

Figure 1. Mobile robot with two independent drive wheels.

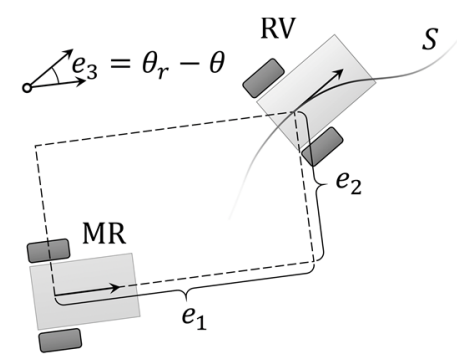

Figure 2. Real mobile robot and referenced vehicle.

If $\mathrm{RV}$ equations of motion are taken in the form

$$
\begin{aligned}
& \dot{x}_{r}=V_{r} \cos \left(\theta_{r}\right) \\
& \dot{y}_{r}=V_{r} \sin \left(\theta_{r}\right) \\
& \dot{\theta}_{r}=\omega_{r},
\end{aligned}
$$

then MR-RV deviation vector can be written as follows 


$$
\left(\begin{array}{l}
e_{1} \\
e_{2} \\
e_{3}
\end{array}\right)=\left(\begin{array}{ccc}
\cos (\theta) & \sin (\theta) & 0 \\
-\sin (\theta) & \cos (\theta) & 0 \\
0 & 0 & 1
\end{array}\right)\left(\begin{array}{l}
x_{r}-x \\
y_{r}-x \\
\theta_{r}-\theta
\end{array}\right) .
$$

Introducing the following change of inputs:

$$
\begin{gathered}
u_{1}=V_{r} \cos e_{3}-\vartheta, \\
u_{2}=\omega_{r}-\omega,
\end{gathered}
$$

and differentiating (2), it gives system (4) after simple calculations [18].

$$
\dot{e}=\left(\begin{array}{ccc}
0 & \omega & 0 \\
-\omega & 0 & 0 \\
0 & 0 & 0
\end{array}\right) e+\left(\begin{array}{c}
0 \\
\sin \left(e_{3}\right) \\
0
\end{array}\right) \vartheta_{r}+\left(\begin{array}{ll}
1 & 0 \\
0 & 0 \\
0 & 1
\end{array}\right)\left(\begin{array}{l}
u_{1} \\
u_{2}
\end{array}\right),
$$

Linearization (4) near $e=0, u=0$ gives (5).

$$
\dot{e}=\left(\begin{array}{ccc}
0 & \omega_{r}(t) & 0 \\
-\omega_{r}(t) & 0 & V_{r}(t) \\
0 & 0 & 0
\end{array}\right) e+\left(\begin{array}{ll}
1 & 0 \\
0 & 0 \\
0 & 1
\end{array}\right)\left(\begin{array}{l}
u_{1} \\
u_{2}
\end{array}\right),
$$

Assuming that $V_{r}(t) \neq 0$ and $\omega_{r}(t) \neq 0$ as $t \rightarrow \infty$, i.e. the mobile robot is not at rest for any $t$, it can be shown that the linearized system (5) is controllable. In particular, it means that there exists a stabilizing control $u$ that continuously depends on the state vector [19]. In [20], this control is proposed to be chosen from the class given by (6).

$$
\begin{gathered}
u_{1}=-k_{1} e_{1}, \\
u_{2}=-k_{2} \operatorname{sign}\left(V_{r}\right) e_{2}-k_{3} e_{3}
\end{gathered}
$$

For known $u_{1}, u_{2}$ and $V_{r}(t), \omega_{r}(t)$ the values of linear $V(t)$ and angular $\omega(t)$ velocities can be easily calculated:

$$
\begin{gathered}
V=V_{r} \cos e_{3}-u_{1}, \\
\omega=\omega_{r}-u_{2} .
\end{gathered}
$$

Thus, the ACS of MR has the structure shown in Figure 3. The following notation is used for this block diagram: $\mathrm{BE}$ is the block for calculating the error vector e according to formula (2), $\mathrm{C}$ is the controller, $\mathrm{BC}$ is the control conversion block in which equations (7) are used to calculate the necessary linear and angular velocities.

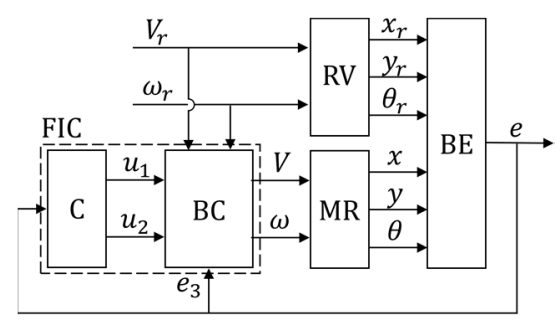

Figure 3. Structure of MR ACS.

\section{Synthesis of the Controller with Constant Coefficients}

A methodology for choosing the coefficients of the controller was proposed in [21]. It makes it possible to stabilize the trajectory motion of MR, in particular, it is shown that the calculation of $k_{i}(i=1,2,3)$ by the formula

$$
\begin{gathered}
k_{1}=2 \xi \sqrt{\omega_{r}^{2}+b V_{r}^{2}} \\
k_{2}=b\left|V_{r}^{2}\right|, \\
k_{3}=2 \xi \sqrt{\omega_{r}^{2}+b V_{r}^{2}},
\end{gathered}
$$

guarantees the local stability of ACS near $e=0$ when $\xi$ and $b$ are positive. However, according to this technique, quality indicators in the synthesis of ACS are not taken into account. Consider the situation when $V_{r}(t) \equiv 1.0, \omega_{r}(t) \equiv 1.0, x_{r}(0)=1, y_{r}(0)=0, \theta_{r}(0)=\pi / 2$, i.e. when a referenced vehicle moves in a unit circle at a constant speed. By substituting the positive values $\xi=1 / 2 \sqrt{2}$, $b=1$ in (8), we obtain the coefficients $k_{i}, i=1,2,3$. The controller (6), (7) with such coefficients 
will be called the controller with constant parameters (CPC). The trajectory motion of the MR with the CPC from the point $x(0)=0, y(0)=0, \theta(0)=0$ is shown in Figure 4 .

The trajectory of the MR with the CPC has significant overshoot and oscillation relative to the trajectory of the RV. The transients of the components of the MR positioning error vector are shown below (Figure 5). It can be seen that the graphs getting into the $5 \%$ zone take about $5.2 \mathrm{~s}$.

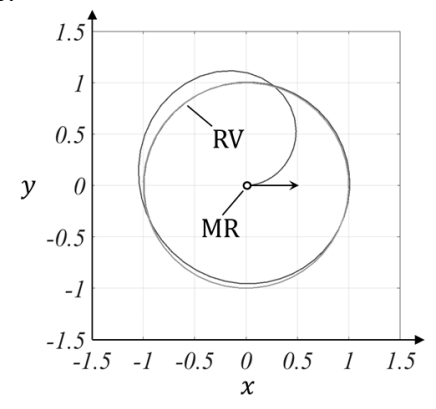

Figure 4. Trajectory of the MR with the CPC.



Figure 5. Error positioning MR with CPC.

\section{Synthesis of the Controller in the Presence of Full Information}

The approximation of differential game value function will be used to synthesize the parameters of the controller in the presence of full information (FIC) about the object state vector. As mentioned in introduction, the computational complexity of numerical methods does not allow to use the approximation of value function in real-time applications. Thus, extensively developed over the last decade mesh-free algorithms will be applied in this work [13, 14]. They are more computationally effective and better cope with the "curse of dimensionality" while theoretical convergence to exact solution is considered in [22, 23]. Moreover, if the value function is positive definite as well as integrand in a performance index, then it is also a Lyapunov function, which guarantees asymptotic stability of the system (for further details see $[15,16])$. So, it permits not only to synthesize stabilizing control laws in the form of feedbacks, but also optimal ones for the chosen integral criterion.

In the problem of trajectory motion of MR, the FIC synthesis method can be used to overcome the difficulty of choosing the coefficients $k_{i}(i=1,2,3)$ in (6), which provide the required quality indicators. In this case, $k_{i}$ will not be constant coefficients as for the CPC, but non-linear functions of the vector $e$. Using information on the physical characteristics of MR (for example, the maximum angular and linear velocities or the maximum voltage and current in the armature windings of electric drives), it is possible to determine the limits of variation of $k_{i}(e)$. The specific nonlinear characteristics $k_{i}(e)$ are determined during the approximation of the value function. The trajectory of the MR with FIC, in which $0 \leq k_{i}(e) \leq 2, i=1,2,3$, and the integrand of the quality criterion has the form $L_{0}(e)=e_{1}^{2}+e_{2}^{2}+e_{3}^{2}$ is shown (Figure 6).

Comparing Figure 4 and Figure 6.a, it can be noted that the FIC provides significantly higher quality indicators of MR movement: overshoot is noticeably reduced, and the oscillation is completely absent. This conclusion is also confirmed by comparing the graphs of the quality criterion for the CPC and FIC with the integrand $L_{0}$. The values of the quality criterion for MR with FIC are two times lower than for MR with CPC as shown in Figure 7. Such comparison clearly demonstrates the superiority of the method proposed in this section for the synthesis of non-linear characteristics of ACS over the approach for the synthesis of CPC from [21]. The nonlinear characteristics of the FIC tuned for the integral criterion with the function $L_{0}$ are presented in Figure 8. Average time for one evaluation of approximated value function is $0.0077 \mathrm{~s}$ ( 2 core $1.9 \mathrm{GHz}$ processor), what must be sufficient for MR real-time control. 


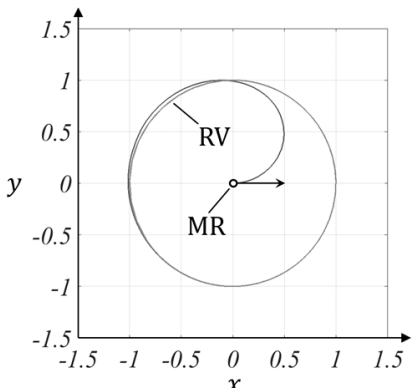

a)



c)

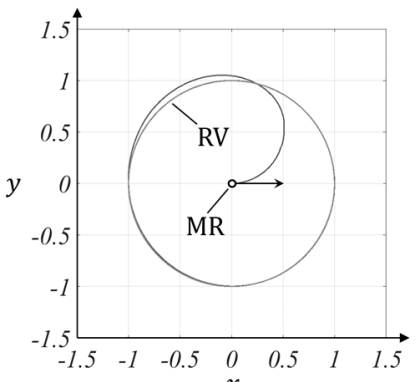

b)

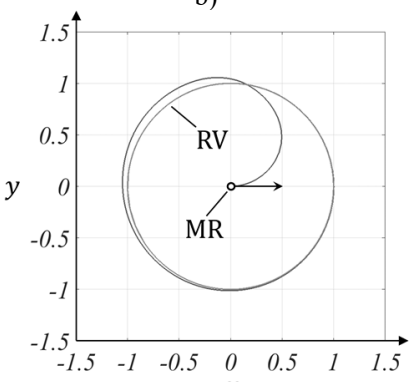

d)

Figure 6. Trajectory of the MR with FIC and various integral criteria: $L_{0}-(\mathrm{a}), L_{1}-$ (b), $L_{2}-(\mathrm{c}), L_{3}-(\mathrm{d})$.

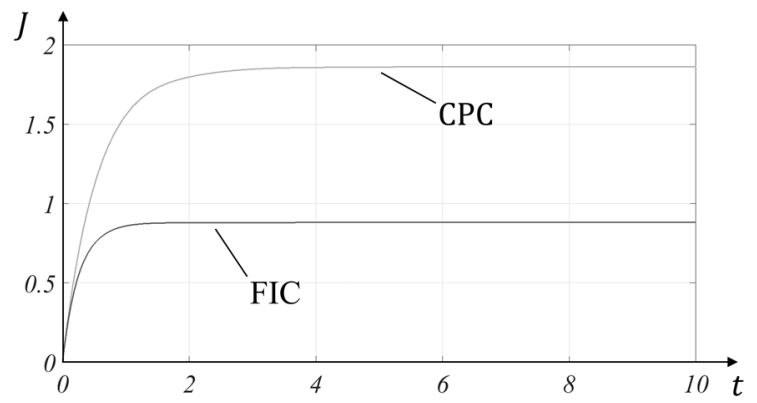

Figure 7. Values of the quality criterion with $L_{0}$ for CPC and FIC.

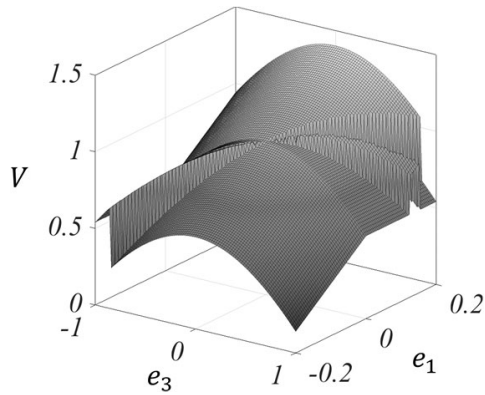

a)

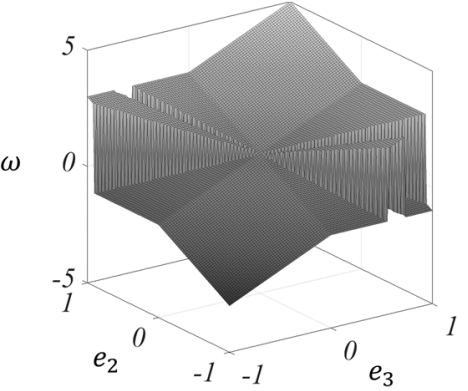

b)

Figure 8. Nonlinear characteristics of FIC: values of the linear velocity $V$ in the slice $e_{2}=0$ (a), values of the angular velocity $\omega$ in the slice $e_{1}=0$ (b). 


\section{Influence of the Integral Criterion on the Quality of the System}

So far, it has been a question of improving the quality of control through the use of a controller that optimizes a specific integral criterion. In this regard, it seems logical to consider the influence of the type of integrand $L$ on such quality indicators as speed, overshoot and oscillation. For this purpose, we consider the motion of MR with various FICs synthesized for several variants of the integrand.

Let's introduce the following functions: $L_{1}(\mathrm{e})=100 e_{1}^{2}+e_{2}^{2}+e_{3}^{2}, L_{1}(\mathrm{e})=e_{1}^{2}+100 e_{2}^{2}+$ $e_{3}^{2}, L_{1}(\mathrm{e})=e_{1}^{2}+e_{2}^{2}+100 e_{3}^{2}$. The graphs of the MR trajectories with the corresponding FICs are shown in Figure 6. As in the case of FIC tuned to the integral criterion with $L_{0}$, these trajectories have less overshoot than MR with CPC (Figure 4), and the oscillation is completely absent. Particular attention should be paid to Figure 6.c, where the movement of MR with FIC is carried out completely without overshoots. This statement is confirmed by the fact that by changing the form of the integral criterion, it is possible to improve the quality of the system. Explanation of this effect in more detail is considered below.

It is easy to see that $L_{j}(j=1,2,3)$ differs from $L_{0}$ in a significant increase in the coefficient for the $j$-th component of the vector $e$ in the integrand. This leads to the fact that the error $e_{j}$ makes a decisive contribution to the final value of the integral criterion with $L_{j}$. Roughly speaking, FIC tuned to the integral criterion with $L_{j}$ selects from all possible MR trajectories the one for which the component $e_{j}$ enters the region close to zero most quickly and with the smallest fluctuations (Figure 9).

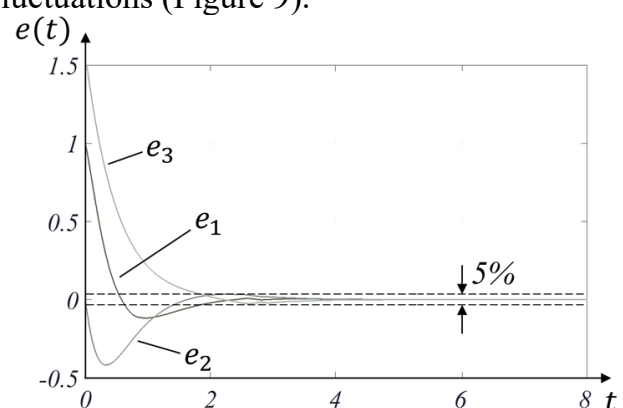

a)

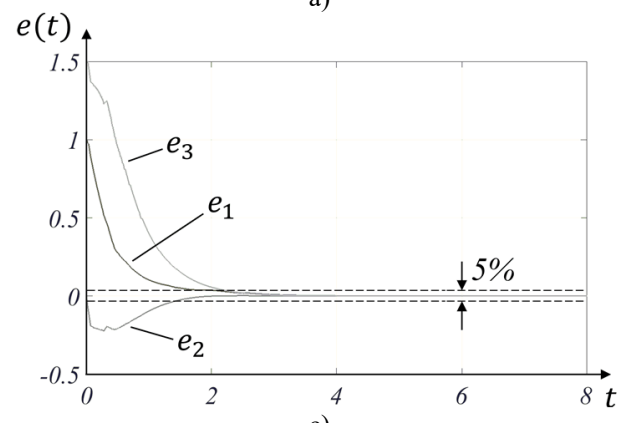

c)



b)

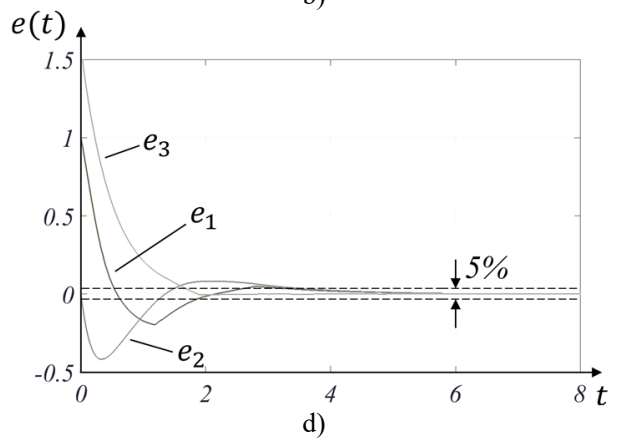

d)

Figure 9. Positioning errors of MR with FIC and various integral criteria: $L_{0}-$ (a), $L_{1}-$ (b), $L_{2}-$ (c), $L_{3}-$ (d).

In view of the above, it is possible to give a physical interpretation to the quality improvement of the control of MR with FIC when using $L_{2}$. Indeed, the fact that the error $e_{2}$ is equal to zero means that the direction of movement of the MR is aimed at the middle of the axis of the driving wheels of the RV. Due to this, overshoots can be avoided, since for their occurrence it is necessary that the center of driving wheels of the RV is located on the left side of the MR movement direction when approaching the trajectory (in the case of counterclockwise 
circular motion of the RV). Since the MR with the FIC tuned to the integral criterion with $L_{2}$ minimizes the error $e_{2}$ to zero as quickly as possible, the approach to the trajectory occurs at the moment when the movement of the MR is already precisely directed to the RV.

The same argumentation can be used as the basis for the conclusion that it is inappropriate to use integral criteria with $L_{1}$ and $L_{3}$. So, the fact that $e_{3}$ is equal to zero means that the directions of motion of the MR and the RV coincide. However, if MR and RV are at different points, then this will more likely increase the transition process. It is clearly seen from the analysis of Figure 9.d. Despite the fact that this controller minimizes $e_{3}$ the fastest, its speed of $3.7 \mathrm{~s}$ is the worst of all others FICs (but at the same time it surpass MR with CPC). A similar situation takes place for the FIC tuned to the integral criterion with $L_{1}$ (Figure 9.b). The fastest possible reduction of the error $e_{1}$ to the minimum values (i.e., to the situation when the $\mathrm{RV}$ is on a straight line passing through the axis of the MR drive wheels) does not guarantee a high-quality transition to the trajectory. In the end of this section it is necessary to note that all approximated value functions and integrands $L_{0}, L_{j}$ are positive definite, so the resulting system is asymptotically stable what can be clearly seen from Figure 9 .

\section{Conclusion}

In the task of trajectory control of a moving object, the FIC provides higher speed, less overshoot and oscillation than the CPC. Another important advantage of FIC is that its non-linear characteristics are synthesized "automatically" when all steps are taken from the corresponding tuning methodology. So, only knowledge of the object model, the capabilities of the control devices (control resource) and the desired integral quality criterion are required. In most cases, obtaining this information is not difficult and can be done before designing ACS. The performed experiments show that by changing the form of the integrand function of the criterion, it is possible to further improve the quality of the system. At the same time, none of the FICs tuned to various integral quality criteria showed lower qualitative characteristics (in terms of speed, overshoot, and oscillation) compared to the CPC.

Since proposed approach has better quality indicators in comparison to classical methods, further development will be directed on physical implementation of control system for real two wheels differentially driven robot. This may require to consider more complex mathematical model of MR, which involves differential equations of higher order as well as presence of external disturbance. In that case the "curse of dimensionality" will play more significant role. To keep evaluation time of approximated value function sufficiently small, some special techniques such as parallelization and sparse grid methods can be applied.

\section{Acknowledgments}

This work was supported by the Russian Science Foundation, according to research project № 16-19-00052. Also, authors would like to thank Natalia Sadivova for translation assistance throughout all aspects of this paper.

\section{References}

1. B. Frank, J. Kleinert, and R. Filla, Automation in Construction. 91, 1 (2018).

2. S. Garnier, K. Subrin, P. Arevalo-Siles, G. Caverot and B. Furet, Procedia CIRP. 72, 297 (2018).

3. N. V. Kumar and C. S. Kumar, Procedia Computer Science. 133, 456 (2018).

4. J. Berg, A. Lottermoser, C. Richter and G. Reinhart, Procedia CIRP. 79, 614 (2019).

5. I. Carlucho, M. De Paula and G. G. Acosta, ISA Transactions. 1 (2020). 
6. S. Li, L. Ding, H. Gao, C. Chen, Z. Liu and Z. Deng, Neurocomputing. 283, 20 (2018).

7. D. Chen and S. Li, Applied Soft Computing. 1 (2019).

8. I. Carlucho, M. De Paula and G. G. Acosta, Expert Systems with Applications. 137, 292 (2019).

9. V. P. Berdnikov, Russian Technological Journal. 6(5), 25 (2018) [In Russ.]

10. V. P. Berdnikov, V. M. Lokhin, S.U. Uvaysov, International Seminar on Electron Devices Design and Production (SED). (2019).

11. V. P. Berdnikov and V.M. Lokhin, Civil Engineering Journal. 5(1), 107 (2019).

12. N. D. Botkin, K.-H. Hoffmann and V. L. Turova, SIAM Journal on Scientific Computing. 33(2), 992 (2011).

13. S. Garg, M. Pant, International Journal of Computational Methods, 15(04), 1830001 (2018).

14. G. Ferretti, R. Ferretti, O. Junge and A. Schreiber, IFAC. 50(1), 1643 (2017).

15. A. L'Afflitto, IEEE Conf. Decision and Control. 2, 1933 (2016).

16. A. L'Afflitto, IET Control Theory \& Applications. 11(15), 2486 (2017).

17. Y. G. Martynenko, Journal of Mathematical Sciences. 147(2), 6569 (2007).

18. A. Bacciotti. Local Stabilizability of Nonlinear Control Systems. World Scientific Publishing Company, 202 p. 1991.

19. G. Cook. Mobile Robots: Navigation, Control and Remote Sensing. Piscataway, NJ: John Wiley \& Sons, 319 p. 2011.

20. W. Dixon, D. M. Dawson, E. Zergeroglu and A. Behal. Nonlinear Control of Wheeled Mobile Robots. Springer, London. 205 p. 2000.

21. C. Samson and K. Ait-Abderrahim, Proc. IEEE/RSJ Int. Workshop on Intelligent Robots and Systems, IROS'91, 1242 (1991).

22. J. Yong, SIAM J. Control Optim. 28(5), 1234 (1990).

23. J. Yong, Journal of Mathematical Analysis and Applicationcs. 145(15), 455 (1990). 